\title{
Water content detection in aviation fuel by using PMMA based optical fiber grating
}

\author{
Wei Zhang ${ }^{1 *}$, David J. Webb ${ }^{2}$, Liyun Lao ${ }^{3}$, David Hammond ${ }^{3}$, Mark Carpenter ${ }^{4}$, Colleen Williams ${ }^{4}$ \\ ${ }^{1}$ School of Aerospace, Transport, and Manufacturing, Cranfield University, MK43, OAL, UK \\ ${ }^{2}$ Aston Institute of Photonic Technology,Aston University, B4 7ET, UK \\ ${ }^{3}$ School of Water, Energy and Environment, Cranfield University, MK43, OAL, UK \\ ${ }^{4}$ Cranfield Defence and Security, Defence Academy of the United Kingdom, SN6 8LA, UK \\ zhang.wei@cranfield.ac.uk
}

\begin{abstract}
Water in aviation fuel is a destructive contaminant and can cause serious problems that compromise aircraft's safe operation and reduce its efficiency and lifetime. Online monitoring of water content in aviation fuel would permit the control of water content before it builds up to dangerous level. Optical fibers made of PMMA have water affinity. In a PMMA based optical fiber Bragg grating (POFBG) its refractive index and volume vary with the water content. This feature is used to detect tiny water content in aviation fuel in this work. The sensing mechanism of POFBG is analyzed. POFBG wavelength is found to be the function of both temperature and equilibrium relative humidity (ERH). POFBG response to water content in fuel can be determined by the ERH. The sensor is experimented at different environmental conditions to identify its sensitivity. As a result, a general expression of POFBG response is achieved. Water content in Jet-A1 is measured by using POFBG sensor calibrated with both environmental chamber and coulometric titration. POFBG sensor is finally tested in a simulation fuel tank, demonstrating a better performance than coulometric titration. A sensitivity of POFBG wavelength change to water content of $33 \mathrm{pm} / \mathrm{ppm}$ is achieved at room temperature, indicating detectable water content of $0.03 \mathrm{ppm}$.
\end{abstract}

Key Words-Fiber Bragg gratings, Polymer optical fiber, Water in fuel, Equilibrium relative humidity; Coulometric titration.

\section{Introduction}

Aviation fuel can hold a certain amount of dissolved water. The maximum amount of water that a given fuel can contain in solution is referred to as its saturation point. Once the fuel has reached its saturation point, any additional water introduced will separate out, in the form of an emulsion of suspended droplets or as a distinct layer. Water contamination in an aircraft system can stem from the fuel containing soluble water which is not removed by filter separators. Humid air in hazardous weather is also transformed to water in the fuel tanks. Even though the amount is small, it is a potential problem since it comes out of solution at low temperatures. This becomes critical when the water content nears the fuel's saturation point, creating a risk of forming free water - a destructive contaminant to almost all fuel applications. In aviation fuel system, water, in addition to not burning in an engine, will freeze at the low temperatures encountered in high altitude flights. The resulting ice may plug fuel filters and otherwise impede fuel flow. Water in fuel system increases risk for lightning damage (due to the water conductivity). It also may facilitate the corrosion of some metals and the growth of microorganisms. Microorganisms found in fuels are very effective at plugging fuel filters. Some microorganisms also generate acidic by-products that can accelerate metal corrosion. The best approach to water contamination is keeping the amount of free water in fuel storage tanks and aircraft fuel tanks as low as possible [1,2]. Monitoring water content in fuel system so to have timely and efficient removal of free water can eliminate the risks.

A typical water-saturated fuel contains between 40 and $80 \mathrm{ppm}$ dissolved water at $21^{\circ} \mathrm{C}$ [3]. This solubility varies with temperature. This makes the measurement task difficult. Free water dispersed in aviation fuel can be detected with a variety of field kits developed over the years by major oil companies. These tests generally rely on colour changes produced when chemicals on a filter go into aqueous solution. The test for undissolved water in aviation turbine fuels has been standardized by American Society for Testing and Materials (ASTM) [4], in which UVilluminated test pad fluoresces when subject to free water in fuel and is compared to a known standard using a photocell comparator. This technique is capable of more precise quantitative results than the chemical tests, although test simplicity is sacrificed. The total water content of aviation fuels (free plus dissolved water) can be measured with the 
ASTM test method by coulometric Karl Fisher (KF) titration [5]. However, this is a laboratory procedure requiring careful sample handling, and results are difficult to compare with the free water tests mentioned above [3].

All currently available dissolved water-in-fuel sensing systems are electrical in nature [e. g., 6, 7] and therefore bring an inherent spark hazard to any fuel tank application. A few optical approaches have been suggested. However, some tend to be based on spectroscopic interrogation of the fuel, which requires an optically clean window to be maintained allowing light to be directed into the fuel and scattered light to be received [8]. Such approaches are not suitable for long term use in a fuel tank where deposits will eventually obscure the window. Another proposed method is sensitive to the refractive index of the fuel rather than water content [9], which introduces ambiguous results. A chem-photonic sensor consisting of an optical fiber with dyed coating was also proposed for one-shot threshold measurement of water content in fuel [10], but this is not suitable for continuous monitoring. There is currently a lack of a convenient, electrically passive system for water-in-fuel monitoring. Instead the airlines rely on colorimetric spot tests or simply draining liquid from the bottom of fuel tanks.

Polymer optical fibers (POFs) have been available for several decades. POFs provide a much lower cost alternative to silica optical fibers, though at a penalty of a much higher transmission loss. As sensors, POFs have additional advantages, including high strain limits, high fracture toughness, high flexibility in bending, large negative thermo-optic coefficients and for some materials an affinity for water. Fiber Bragg gratings have been inscribed into step index and microstructured POF [11] with fast inscription technique [12]. One of the most common materials for POFs is poly (methyl methacrylate) (PMMA). The affinity for water in PMMA leads to a swelling of the fiber and an increase of refractive index, both of which contribute to an increase in the Bragg wavelength of a POFBG [11]. POFBG therefore is considered as a good candidate for quantifying the small amount of water present in aviation fuel.

\section{Principle}

\subsection{Water solubility in fuel}

In the absence of free water, jet fuel can pick up water from the air. Water dissolving in fuel from the surrounding air obeys Henry's law stating that the solubility of a gas in a liquid is directly proportional to the partial pressure of the gas above the liquid. The amount depends on the relative humidity of the air. However, each fuel has its specific water-saturation point beyond which excess water comes either emulsified or free. The equation below provides the estimation for water solubility in fuel at the specified condition (mass \%) [1]:

$$
S=S_{0} H\left(\frac{P}{P_{0}}\right)\left(\frac{T}{T_{0}}\right)^{a}
$$

where $S_{0}$ is the maximum solubility of water in the fuel at $100 \%$ relative humidity, $P_{0}=0.1 \mathrm{MPa}$, and $\mathrm{T}_{0}=293 \mathrm{~K}, P$ the air pressure above the fuel, $T$ the temperature of the air and fuel, $H$ the relative humidity of air above the fuel, $a$ the constant depending on the fuel. At the specified temperature and air pressure the water solubility is proportional to the environmental humidity. Fig. 1 shows the water solubility, $S_{0}$, in Jet-A1 aviation fuel at different temperature.

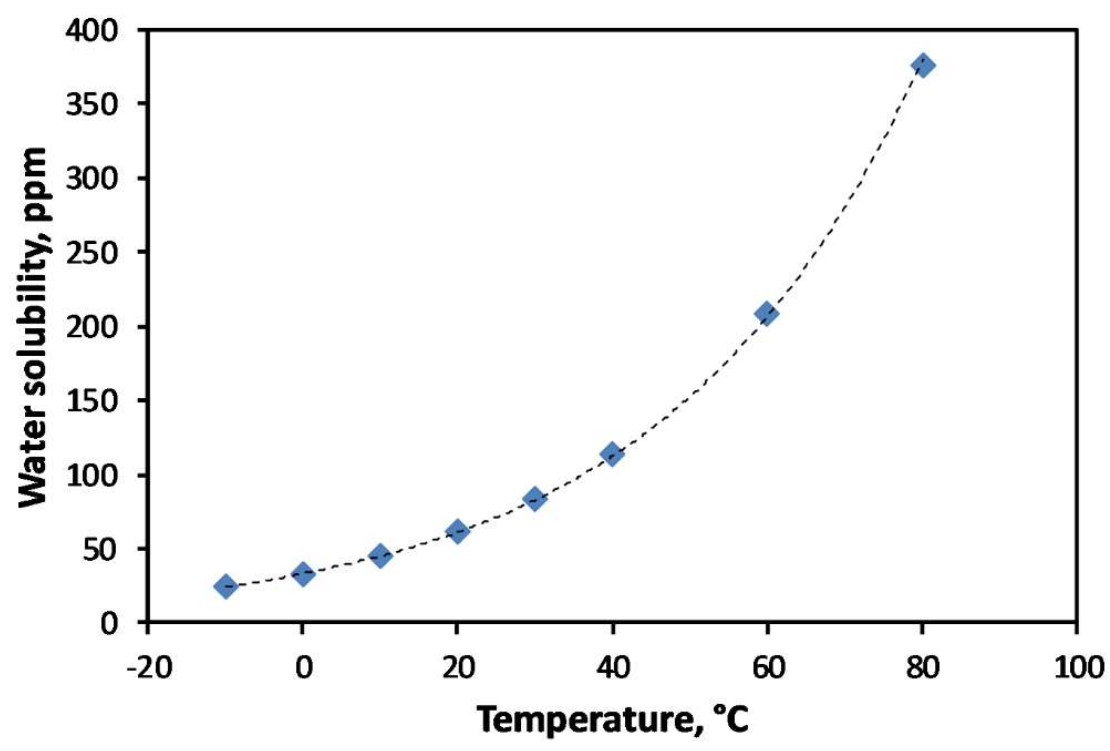

Fig. 1 Water solubility in Jet-A1 


\subsection{PMMA water absorption}

Water absorption introduces changes in volume and refractive index of PMMA. The dependence of PMMA refractive index on relative humidity has been measured at different temperature [13]. Water absorption in PMMA derived in the multimolecular theory of absorption, can be expressed as,

$$
w=\frac{w_{1} x\left(1-6 x^{5}+5 x^{6}\right)}{(1-x)\left(1-x^{6}\right)}
$$

where $w$ is the weight of absorbate per gram of adsorbent, $w_{1}$ the weight of absorbate per gram of adsorbent when each adsorption site is covered by one mol. of adsorbent [14], $x$ the ratio of the equilibrium absorption pressure to the saturation pressure over a free liquid surface of the absorbate, representing the equilibrium relative humidity (ERH). Here the absorbate is water and the absorbent PMMA. For the PMMA, $w_{l}$ is 6.25 .

According to (2) water absorption in PMMA is a function of ERH. Considering a unit volume of an initially dry polymer, of density $\rho_{0}$, which takes up $w$ wt $\%$ water, the density change of PMMA against water uptake rate can be calculated as [14],

$$
\rho=\rho_{0} \frac{1+w / 100}{1+w f \rho_{w} / 100}
$$

where $f$ is a fraction of the water contributes to an increase in the PMMA volume, $\rho_{w}$ the density of water. The volume change of PMMA caused by humidity can be estimated by using (2) and (3), and the related swelling coefficient along the fiber direction which usually is a third of the volumetric change, can be expressed as,

$$
\beta=\frac{\Delta V}{3 V_{o}}=\rho_{w} f w / 300
$$

\subsection{PMMA optical fiber Bragg grating}

Bragg wavelength of a fiber grating depends on the effective core refractive index $n_{\text {eff }}$ and the grating pitch $\Lambda$. From the above analysis one can expect that both parameters in a POFBG can be modulated by the temperature $T$ and water content inside the grating or surrounding relative humidity $H$,

$$
\lambda_{B}=2 n_{e f f}(T, H) \Lambda(T, H)
$$

For constant temperature the wavelength change of POFBG against humidity can be expressed as

$$
\Delta \lambda_{B}=\lambda_{B}(\eta+\beta) \Delta H
$$

where $\lambda_{B}$ is initial Bragg wavelength, $\eta$ is the normalized refractive index change on humidity $(\% \mathrm{RH})^{-1}, \beta$ the swelling coefficient related to humidity induced volumetric change $(\% \mathrm{RH})^{-1} . \eta$ is known though it is temperature dependent [13]. $\beta$ can be determined from the PMMA volumetric change induced by water absorption, defined by (4), which is temperature dependent, too [15]. As a result, the wavelength change in POFBG indicates the change of relative humidity at each temperature. When POFBG is placed in aviation fuel, its wavelength change reflects the water content in the fuel which is directly proportional to the relative humidity of air above fuel once the fuel-air system has reached equilibrium.

\section{Experiment}

The amount of dissolved water in fuel depends on the relative humidity of the air above the fuel as shown by (1). Henry's law indicates that the water in fuel reaches equilibrium with free water or moist air. Fuel close to a fuel-water or fuel-air interface will reach water equilibrium in a matter of minutes. Therefore, the water content can be induced to fuel by exposing fuel to the air. Different amounts of water in fuel can be adjusted by changing the surrounding relative humidity. When in equilibrium water in fuel is a function of ERH.

An experiment arrangement was set to simulate the induced water in fuel and investigate the performance of POFBG for water detection in fuel, as shown in Fig. 2. Jet-A1 aviation fuel was held in a beaker placed in an environmental chamber (Sanyo Gallenkamp) to be in contact with the air that can be set to different temperature and humidity. POFBGs were fabricated in a short length of PMMA optical fiber and attached to a single mode silica fiber down-lead using UV curable glue [16]. POFBG was inserted into the fuel and monitored under different conditions. A typical POFBG reflection spectrum is shown in the inset of Fig. 2. In this work the wavelengths of POFBGs were monitored by using an IBSEN I-MON 400 wavelength interrogation system. 


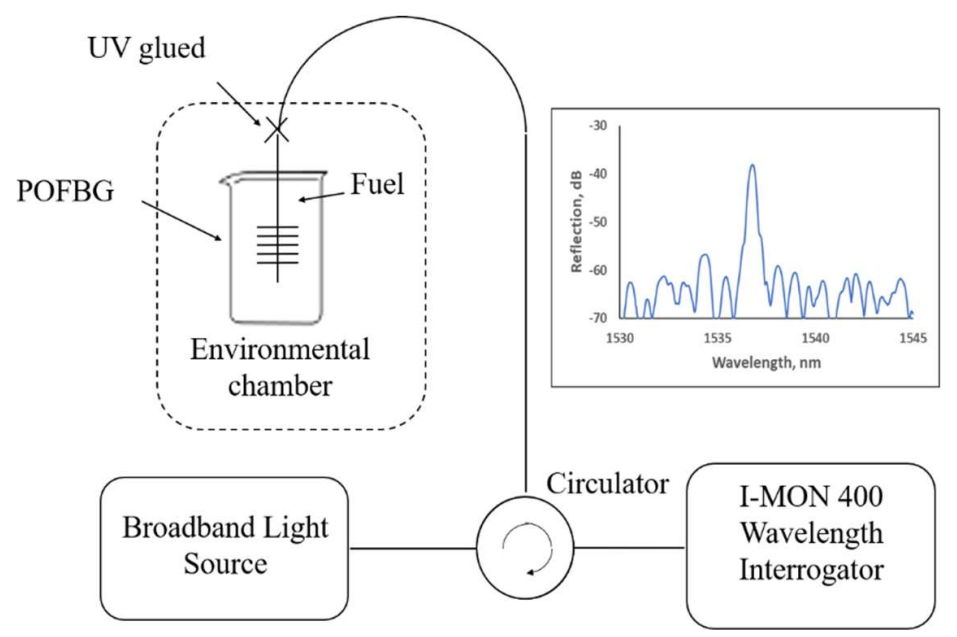

Fig. 2 Experimental arrangement

\subsection{POFBG humidity response at constant temperature}

First the temperature of the environmental chamber was set constant at $24.5^{\circ} \mathrm{C}$ and the relative humidity was programmed for step change. Since it takes time for fuel to reach water equilibrium the chamber was kept at each humidity value for long time (up to 4 hours for each humidity setting). The measured wavelength response of POFBG in fuel is shown in Fig. 3 in which the relative humidity is step changed from $45 \%, 60 \%, 75 \%$, to $90 \%$. One can see that for each humidity setting the POFBG response takes long time to reach a stable value. This long response time is attributed to the time of water equilibrium in the fuel as the response time of POFBG to direct humidity change is much faster [16].

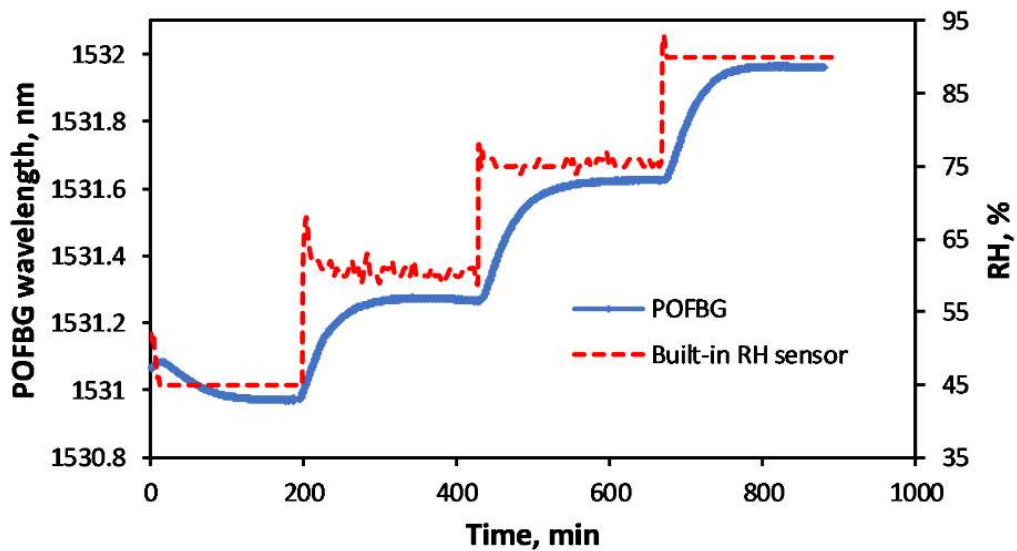

Fig. 3 Response of POFBG inserted in fuel over humidity change at fixed temperature shows long equilibrium time

The equilibrium time closely depends on the volume and the container of the fuel. If the volume of fuel is large and the area of the interface between fuel and air is limited it will take much longer to reach water equilibrium. To accelerate the water equilibrium process in the fuel a magnet stirrer was used to stir the fuel in the beaker. The wavelength response of POFBG in stirred fuel was monitored at different relative humidity. Fig. 4 shows a typical response of the POFBG sensor recorded while the humidity was changed in a step of $10 \%$ from $40 \%$ to $90 \%$ and the chamber temperature was kept at $24.5^{\circ} \mathrm{C}$. The response of POFBG in the air of chamber is plotted in Fig. 4 , too. 


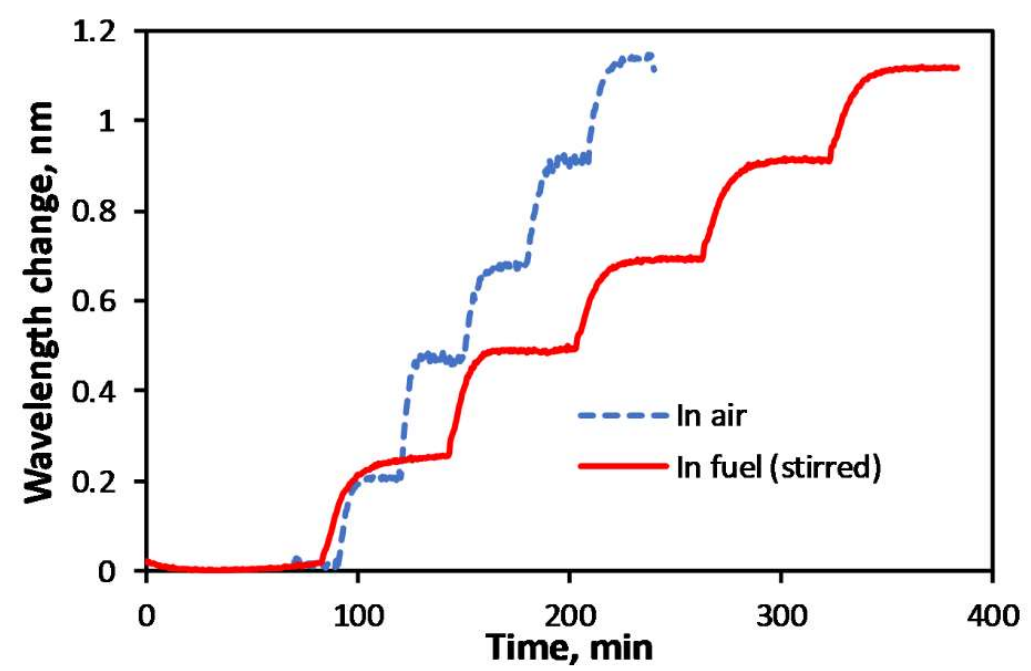

Fig. 4 Responses of POFBG in air and in stirred fuel over humidity change at fixed temperature

A comparative illustration of the time responses of the sensor in three different cases is shown in Fig. 5 where the relative wavelength changes of the POFBG over a $10 \%$ relative humidity step change are plotted. A response time of 65 minutes was recorded when the POFBG was placed in the fuel (no stir). The response time was significantly reduced to 19 minutes when the fuel was stirred by a magnet stirrer. When the POFBG was placed in the air of the chamber it showed a response time of $\sim 10$ minutes. This is the response time of POFBG for humidity sensing which is dominated by the time for water diffusing into the fiber core [16]. This time can be much faster by further reducing the size of POF [17]. The time to establish step humidity change in chamber is negligible compared to that.

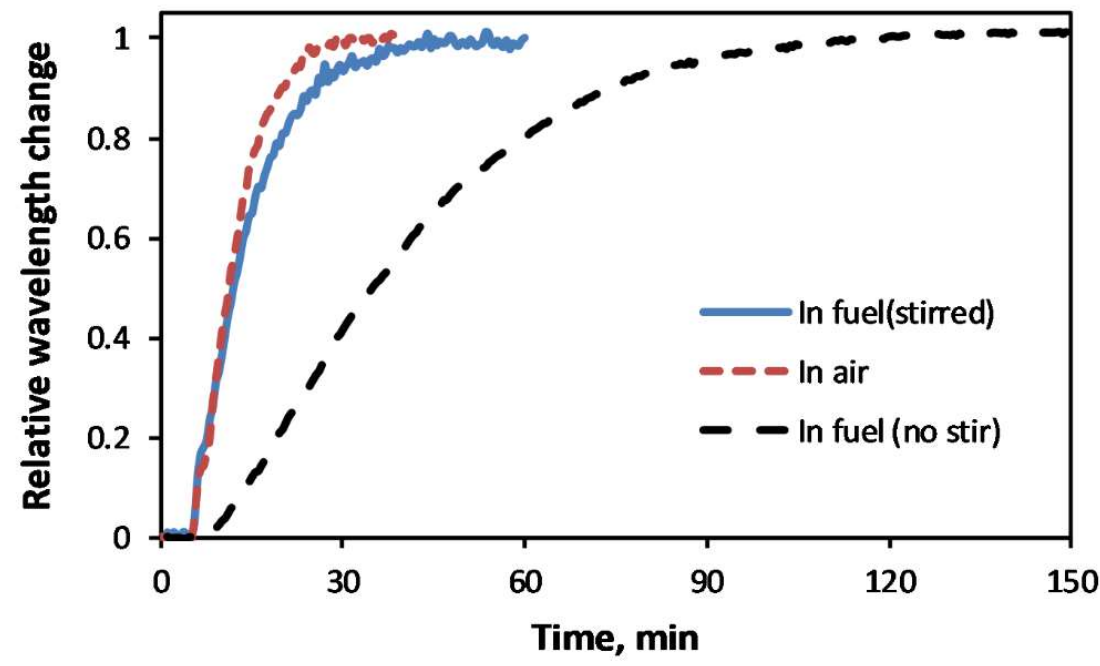

Fig. 5 Responses of POFBG in air, fuel, and stirred fuel over 10\% humidity step change

\subsection{POFBG response as a function of humidity and temperature}

The response of a POFBG consists of the contributions of both temperature and humidity. In (6), however, both $\eta$ and $\beta$ are temperature dependent. The humidity sensitivity of POFBG varies with the surrounding temperature. This makes POFBG response more complicated than silica optical fiber Bragg grating (SOFBG) response in which strain/temperature dual sensitivity can be expressed by a $2 \times 2$ matrix. The POFBG needs to be examined at different settings of humidity and temperature to find a general expression of response in the interested operation range.

An SOFBG can be used to determine the temperature in fuel. Since silica optical fiber exhibits no humidity sensitivity [18] the wavelength of SOFBG only responds to the temperature change in the fuel. Experiments were 
carried out to measure the wavelength responses of SOFBG and POFBG, both inserted in the fuel, against both humidity and temperature change. Due to limited operational range of the environmental chamber the temperature was set from $15^{\circ} \mathrm{C}$ to $25^{\circ} \mathrm{C}$ and the relative humidity was step changed from $50 \%$ to $90 \%$. The chamber dwelled at each setting for 2 hours so to allow the ERH fully established in fuel. Therefore, the applied relative humidity eventually is equal to the ERH. The measured wavelength responses of the POFBG and SOFBG in fuel are summarized in Fig. 6.

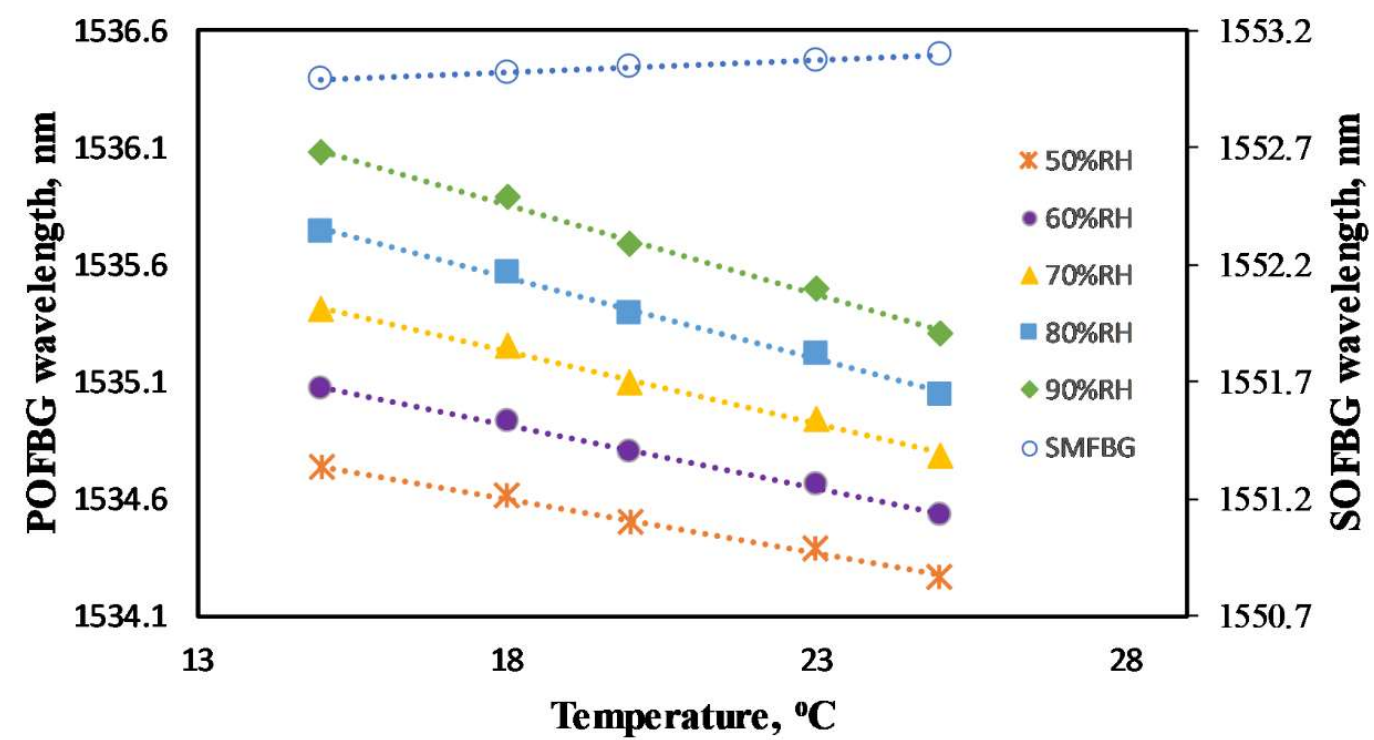

Fig. 6 Responses of POFBG in fuel against humidity change at different temperature

One can see that POFBG response is the function of both temperature and relative humidity. However, the SOFBG response varies only with temperature. Once the temperature is identified by SOFBG the humidity response of POFBG can be determined.

According to the measured results the wavelengths of both POFBG and SOFBG can be expressed as a function of humidity and/or temperature:

$$
\begin{gathered}
\lambda_{\text {POFBG }}=1536-0.1073 T+2.7 \mathrm{H}-0.0217 \mathrm{HT} \\
\lambda_{S M F B G}=0.0102 \mathrm{~T}+1550.18
\end{gathered}
$$

Note that the coefficients in (7) and (8) have different units corresponding to thermal expansion $\left(\mathrm{nm} /{ }^{\circ} \mathrm{C}\right)$, swelling $(\mathrm{nm} / \% \mathrm{RH})$, and the synergic effect $\left(\mathrm{nm} /{ }^{\circ} \mathrm{C} \cdot \% \mathrm{RH}\right)$.

Alternatively, a lookup table for the sensor can be established to find the ERH level once the temperature is decided by the SOFBG response. When the POFBG sensor operates within the calibrated range the ERH in fuel can be calculated by using the above equations to estimate the water content in fuel.

\subsection{POFBG responses in a simulation fuel tank}

A simulation fuel tank rig was set up at Cranfield University for ice accretion and dislodge laboratory tests. The schematic of the test rig is as shown in Fig. 7. The fuel temperature can be reduced by the cooling plates located at the bottom of the tank. The warm water bath can increase the fuel temperature and hence increase the fuel water absorption. The recirculating pump introduces condition changes like flow, vibration and pressure. 


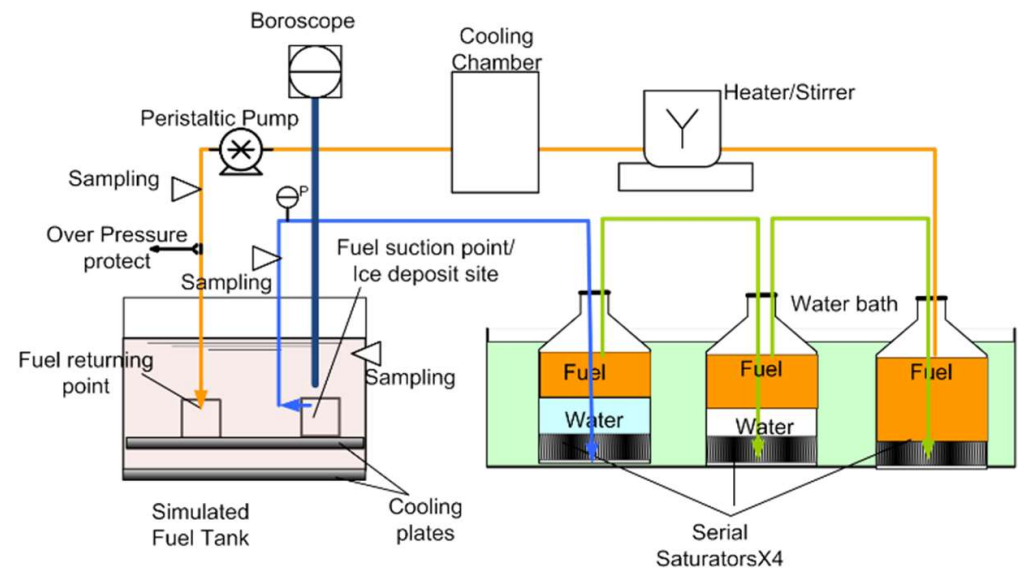

Fig. 7 Schematic of the Bench Test Rig

An optical water in fuel sensor prototype consisting of one POFBG and one SOFBG, was deployed for test in the simulation fuel tank. FBGs were inserted into the fuel tank at increasing temperature starting from $10^{\circ} \mathrm{C}$ (this is the ambient temperature in December while good ventilation maintains for the site). Since the dissolved water in fuel varies with the temperature the POFBG sensor was expected to respond to both changes in temperature and water content.

The fuel temperature was monitored by using both SOFBG and thermocouples. Several thermocouples were placed in the simulation fuel tank to monitor the temperature distribution in fuel. The temperature reading from the thermocouple closest to the SOFBG was adopted. Measured responses of both POFBG and SOFBG in the fuel while temperature varied from $10^{\circ} \mathrm{C}$ to $25^{\circ} \mathrm{C}$, are shown in Fig. 8 .

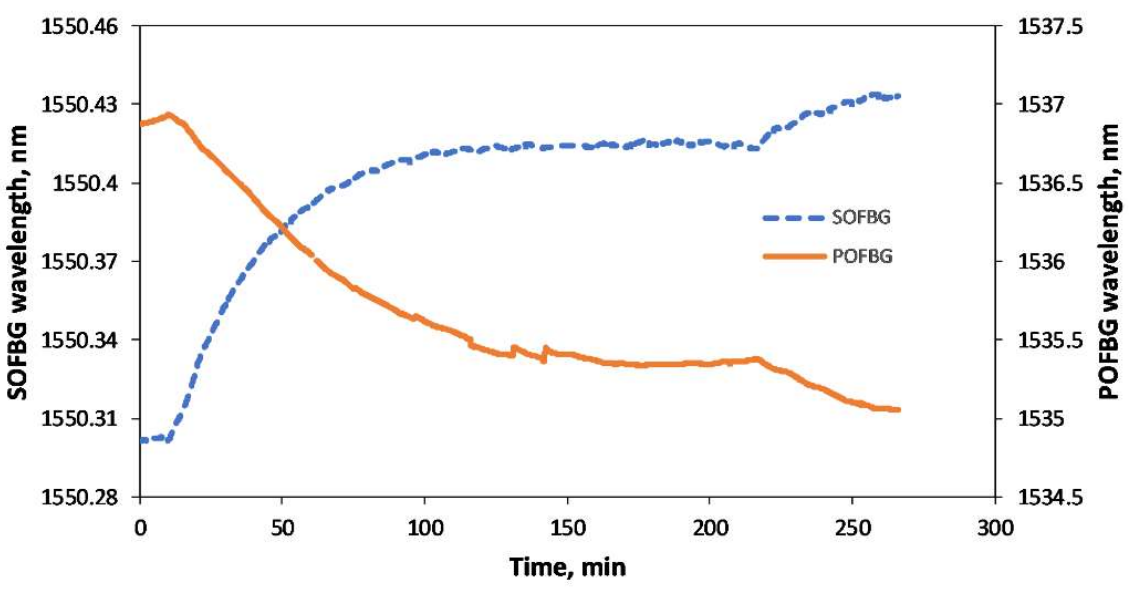

Fig. 8 Measured responses of optical water in fuel sensor at test

A KF coulometer was used to measure the water content in fuel samples at different temperatures. Three fuel samples were taken from the simulation fuel tank at each of chosen temperatures. Only the readings of three measurements having diversity less than $\pm 15 \%$ were selected and averaged for estimating the water content in fuel. The measurements of KF coulometer showed a considerable diversity. This is because errors can be readily induced during sample handling in the procedure of KF coulometric titration.

Fig. 9 shows the measured water contents by using KF coulometer during the test where the temperature measured by using thermocouple and SOFBG (its wavelength response converted to temperature by using (8)) are plotted there, too. 


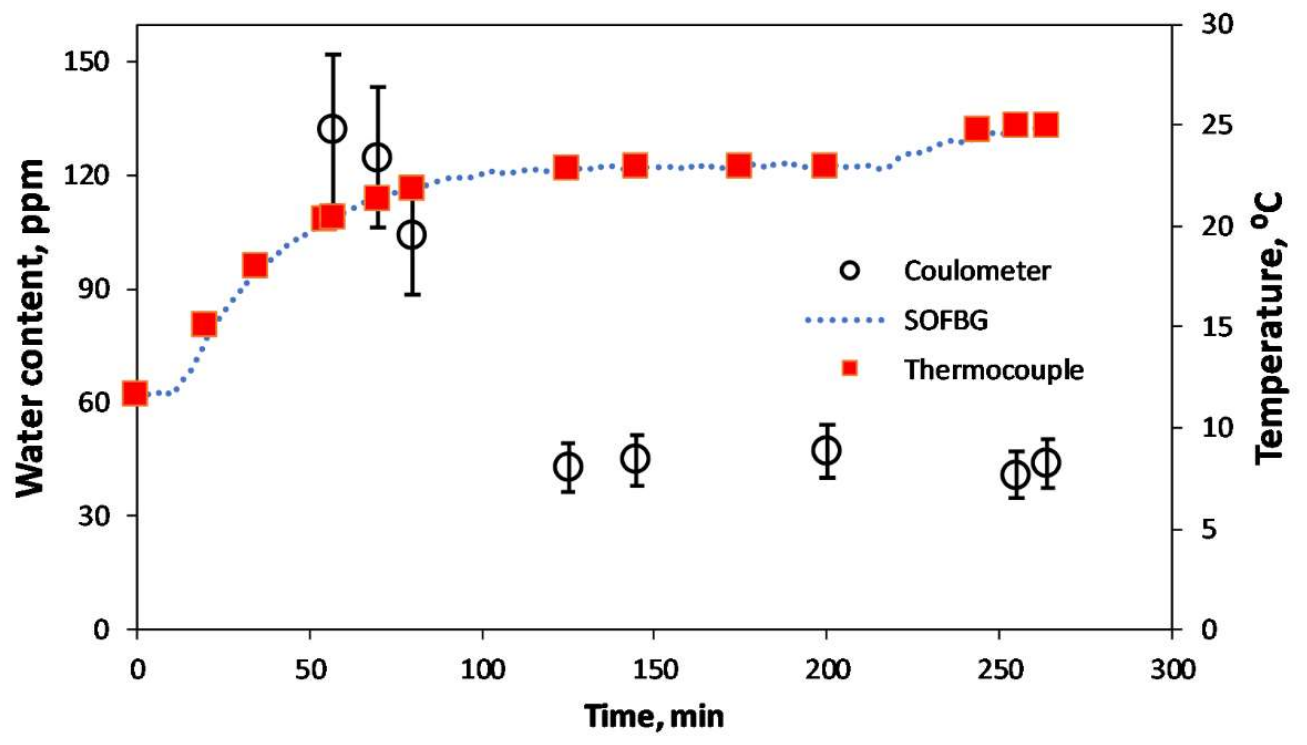

Fig. 9 Measured responses from KF coulometer and thermocouple. SOFBG response was expressed in ${ }^{\circ} \mathrm{C}$ as a comparison.

The POFBG response is not self-evident due to its dependence to both temperature and humidity. When temperature increases, the water solubility rises, leading to more water dissolved in the fuel. The SOFBG response shows a very good agreement with the thermocouple reading. Once temperature known one can extract humidity information from POFBG response. In the following section, we will analyze all the results from fiber gratings, thermocouple and KF coulometer, to get an insight into the POFBG performance.

\section{Analysis and discussion}

The water solubility of Jet-A1 at the corresponding temperature is calculated and plotted in Fig. 10 together with the water content measured using KF coulometer. The first three measured water content values are larger than the corresponding solubility, indicating that there is free water or emulsion in the fuel.

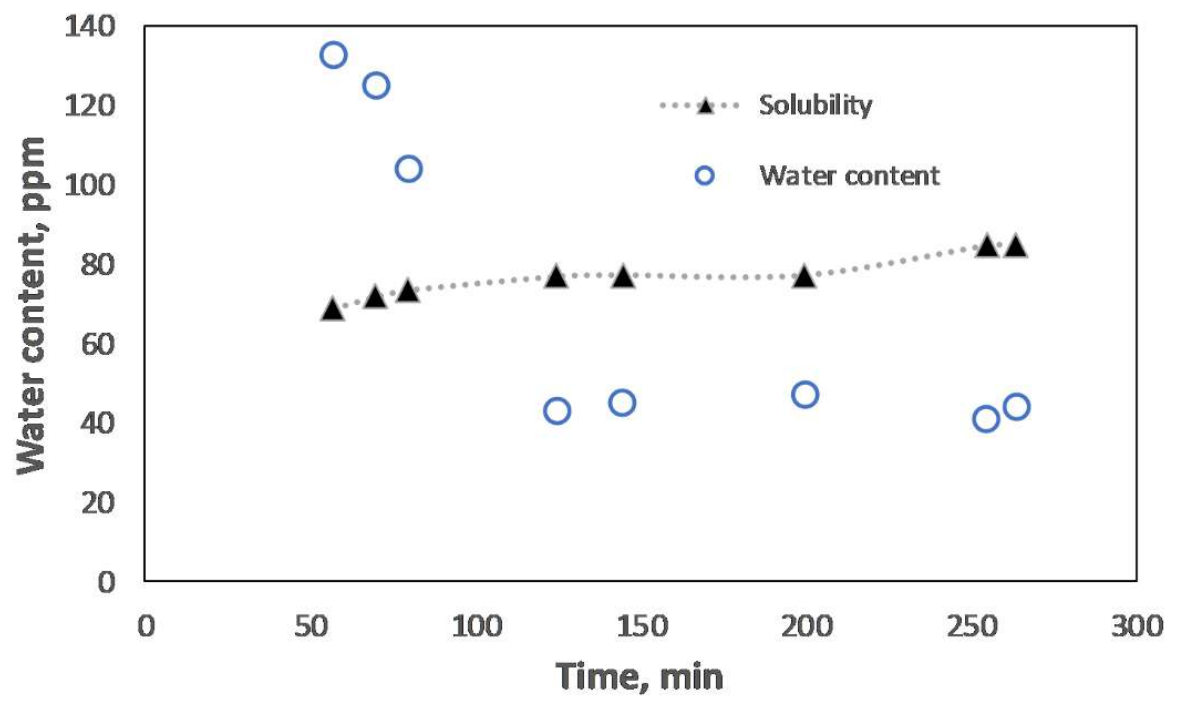

Fig. 10 Measured water content in fuel by KF coulometer and its solubility at different temperatures

POFBG response is a function of ERH and temperature. It was calibrated by using the environmental chamber. At a specified temperature the ERH can be calculated using (7) from the measured POFBG response: 


$$
E R H=\left(1536-\lambda_{P O F B G}-0.1073 T\right) /(0.0217 T-2.7)
$$

From the measured POFBG response shown in Fig. 8 we can estimate the ERH in fuel by using the above equation at the temperatures where the fuel samples were analyzed by KF coulometer. Fig. 11 shows the ERHs of the fuel from the measured results by KF coulometer and POFBG sensor. Note that ERH in fuel is equivalent to the ratio of measured water content to solubility at each temperature [1]. In the case of free water or emulsion ERH is considered as unity.

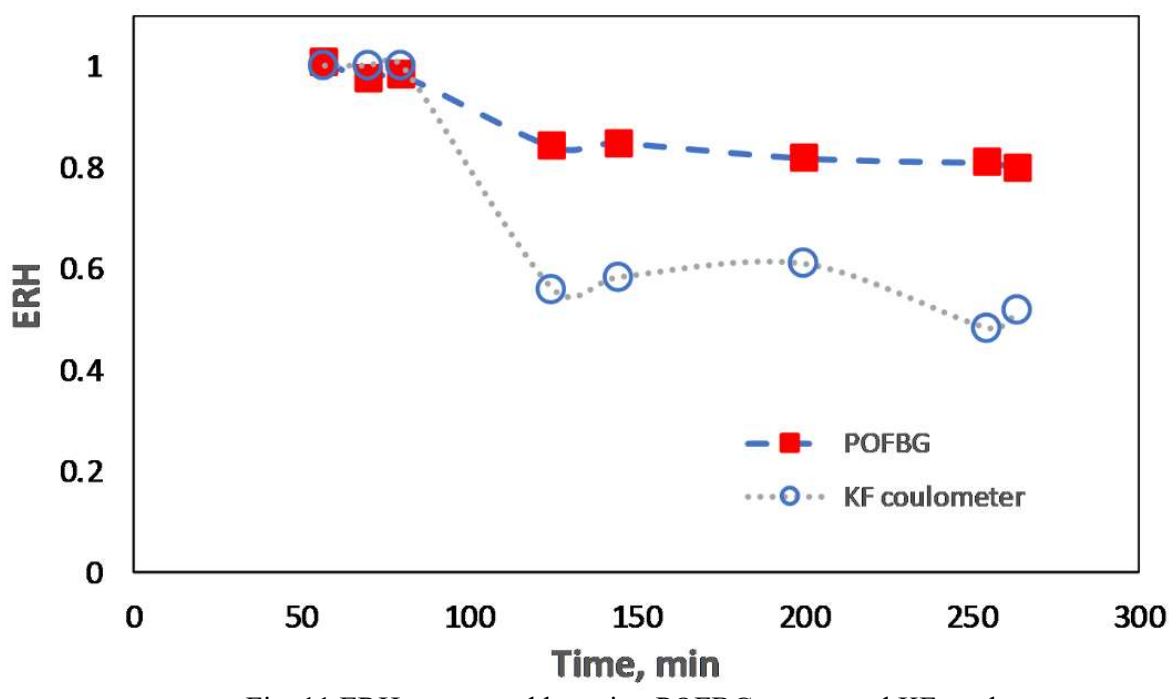

Fig. 11 ERHs measured by using POFBG sensor and KF coulometer

From Fig. 11 one can see that the results are not in good agreement with each other even though they show similar tendency. Apart from the first 3 values where the water contents are saturated at the temperatures, the measured results from KF coulometer are smaller than that measured by POFBG. This may largely attribute to the procedure of using KF coulometer. In fact, three samples at each temperature might contain different water contents even though they are taken at almost the same time and temperature. Changes may occur during sample handling due to different ambient temperature/relative humidity and analyzing time. This is the reason that three samples show a considerable diversity themselves. When a sample is taken from the fuel tank, before it is analyzed it tends to get equilibrium with ambient environment $\left(\sim 10^{\circ} \mathrm{C}, 50-60 \% \mathrm{RH}\right)$. In this case it leads to reduced measurement of ERH by KF coulometer. In fact, $\mathrm{KF}$ coulometer is prone to a high level of error and its results are often difficult to interpret even though it can resolve ppm water content. This method is not a real-time one and difficult for on-site measurement. From the results in Fig. 11 it seems that the result from POFBG is more credible. If following precise procedure and operated in laboratory, however, KF coulometer can still provide a good measurement for tiny water content.

Further experiment was designed to verify the POFBG sensor performance. Three samples of fuel were carefully prepared. The fuel was dispensed into three glass vials. The vials were uncapped during the conditioning period. The samples were conditioned for 6 days at ambient temperature which varied slightly over the conditioning period, but averaged $20^{\circ} \mathrm{C}$. Sample 1 was made by placing the vial in a desiccator which contained a bed of silica gel. Sample 2 was left open and exposed to ambient air. Sample 3 was exposed to a $100 \% \mathrm{RH}$ atmosphere by placing the vial in a desiccator that contained a small amount of distilled water. On conclusion of the conditioning period, the water content of each sample of fuel was measured using a KF coulometer. Since the sample was measured at the same environment as conditioning, there is no significant diversity in the coulometer measurement. The measurements were recorded as sample 1 of $10 \mathrm{ppm}$, sample 2 of $39 \mathrm{ppm}$, sample 3 of $68 \mathrm{ppm}$. Converting these water contents into ERHs at $20^{\circ} \mathrm{C}$ gives $14.8 \%, 57.8 \%$ and $100 \%$, respectively.

A vial cap was drilled to allow the POFBG put through. Then the samples were tested at the ambient temperature of $\sim 20{ }^{\circ} \mathrm{C}$ by inserting the POFBG into the vial and the vial was then sealed. In this case the water content in the vial maintained unchanged. 


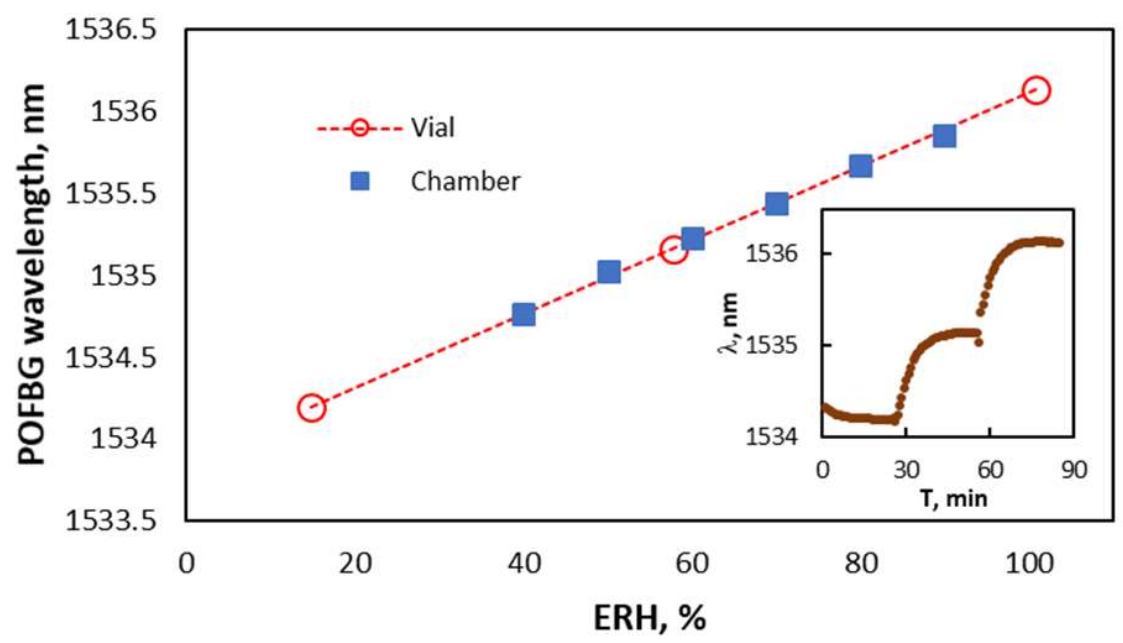

Fig. 12 POFBG responses for the water contents in 3 samples and the fuel conditioned in environmental chamber show good agreement. The inset shows the recorded POFBG response over 3 samples.

The POFBG responses against the water contents in the three samples were measured and are plotted in Fig. 12 where its responses against water contents at the same temperature and different ERH conditioned in environmental chamber were measured and are plotted as a comparison. It indicates that the POFBG sensor can measure the water content in fuel in good agreement with KF coulometer if precise procedure for KF titration is maintained; both results can be represented by (9).

The sensitivity of water content detection in fuel using the POFBG can be estimated as $\sim 33 \mathrm{pm} / \mathrm{ppm}$ at $20^{\circ} \mathrm{C}$. The wavelength resolution for the wavelength interrogation system used in this work is $\sim 1 \mathrm{pm}$. This suggests that the minimum detectable water content in fuel is as good as $0.03 \mathrm{ppm}$ at this temperature. This is a much better performance than any available water content detection instruments.

In the defined range the POFBG response can be well approximated by (7). However, (7) cannot be used to express POFBG response when free water or emulsion exists in fuel. This largely explains the difference for the first three measurements in Fig. 11 where the water content was saturated in the fuel. Also, it is not expected to express the POFBG response in an extended range without having calibrated the sensor.

\section{Conclusion}

In this work we have intensively investigated POFBGs for detecting water content in aviation fuel. A prototype of optical water in fuel sensor based on POFBG has been developed and tested in both environmental chamber and simulation tank. The sensor demonstrates good performance over the fuel samples conditioned by using environmental chamber. It has been justified to some extent by using specialized equipment such as coulometer. The POFBG response exhibits the similar tendency of water content change as KF coulometer does but there is considerable disagreement. Our analysis and experiment have shown that this may be caused by the sample handling in the procedure of onsite KF titration. However, the POFBG sensor measurement shows a good agreement with that obtained in laboratory by careful operation of KF coulometer. The POFBG sensor has shown a high sensitivity in detecting the water content in aviation fuel.

One of our concerns is that the POFBG sensor has been tested only in a limited temperature range in both environmental chamber and simulation fuel tank. Calibration against POFBG in an extended range is difficult. In a low temperature environment water content in fuel generally becomes saturated and the POFBG response reaches a maximum ERH. The explosive and volatile nature of aviation fuel prevents both environmental chamber and simulation tank from setting fuel sample at high temperature. To fully justify the POFBG sensor performance a matured onsite precise measurement technique is needed which currently is not feasible. More samples of fuel with different water contents calibrated by using KF coulometer will be prepared for verifying POFBG sensor performance in a wider environmental condition. Another concern is that dissolved water in fuel takes time to get equilibrium. Larger volume of fuel means longer equilibrium time. Therefore, a real time, distributed/multipoint sensor with precise measurement is demanded for monitoring water content in fuel. POFBG sensor has shown the potential in real time 
monitoring of water content in fuel. The distributed/multipoint sensing can be realized by wavelength-divisionmultiplexing many POFBGs in fuel tank. For this purpose, POFBGs need to operate in low loss wavelength band (around $850 \mathrm{~nm}$ ) rather than current wavelength band (around $1550 \mathrm{~nm}$ ) [11]. This will be our next work.

\section{Acknowledgement}

The research leading to these results has received funding from the European Union Seventh Framework Programme under grant agreement no. 314032. 


\section{References}

[1] Handbook of aviation fuel properties. CRC Inc. 1983

[2] Aviation fuels technical review (www.cgabusinessdesk.com/document/aviation_tech_review.pdf)

[3] Salvatore J. Rand, Significance of Tests for Petroleum Products, 7th ed. ASTM International.

[4] ASTM D3240-15 Standard Test Method for Undissolved Water In Aviation Turbine Fuels, ASTM International, West Conshohocken, PA, 2015, https://doi.org/10.1520/D3240-15

[5] ASTM D6304-16e1 Standard Test Method for Determination of Water in Petroleum Products, Lubricating Oils, and Additives by Coulometric Karl Fischer Titration, ASTM International, West Conshohocken, PA, 2016, https://doi.org/10.1520/D6304-16E01

[6] W. J. Lewis, Water Detection Device for Fuel Line, US Patent, 3,787,650 (1974)

[7] Kaida Khalid, Ionel Valeriu Grozescu, Lim Keng Tiong, Lee Teck Sim and Roslim Mohd, "Water detection in fuel tanks using the microwave reflection technique," Meas. Sci. Technol., 14,1905-1911 (2003)

[8] Yuliya Luzinova, Bogdan Zdyrko, Igor Luzinov and Boris Mizaikoff, "Detecting trace amounts of water in hydrocarbon matrices with infrared fiberoptic evanescent field sensors",

Analyst, 137, 333-341, 2012,

[9] Sean D. Pucketta and Gilbert E. Pacey, "Detection of water in jet fuel using layer-by-layer thin film coated long period grating sensor," Talanta, 78,1,300-304 (2009)

[10] M. Farries, N. Hayes, J. Kelley, G. Tippet, D. Webb, B. Tighe, K. Zhou, D. Harvey, "Optical methods for detecting contamination in jet fuel", Avionics, Fiber-Optics and Phototonics Technology Conference, 2009.

[11] D. J. Webb and K. Kalli, "Polymer Fiber Bragg Gratings," in Fiber Bragg Grating Sensors: Thirty Years from Research to Market, A. Cusano, A. Cutolo, and J. Albert eds, New York: Bentham Science Publishers Ltd, 2010.

[12] C. A. F. Marques, A. Pospori, G. Demirci, O. Çetinkaya, B. Gawdzik, P. Antunes, O. Bang, P. Mergo, P. André and D. J. Webb, "Fast Bragg Grating Inscription in PMMA Polymer Optical Fibres: Impact of Thermal Pre-Treatment of Preforms," Sensors, 17(4), 891, 2017.

[13] Toshio Watanabe, Naoki Ooba, Yasuhiro Hida, and Makoto Hikita, "Influence of humidity on refractive index of polymers for optical waveguide and its temperature dependence", Appl. Phys. Lett., 72, 1533, (1998).

[14] A. M. Thomas, "Moisture Permeability, Diffusion and Sorption in Organic Film-Forming Materials," Journal of Applied Chemistry, 1, 141, (1951).

[15] D. T. Turner, "Polymethyl methacrylate plus water: sorption kinetics and volumetric changes," Polymer, 23, $197-$ $202(1982)$

[16] W. Zhang, D. J. Webb, G.-D. Peng, "Investigation into Time Response of Polymer Fiber Bragg Grating Based Humidity Sensors,” IEEE J. Lightwave Tech 30(8), pp. 1090 - 1096, 2012

[17] Ginu Rajan, Yusof Mohd Noor, Bing Liu, Eliathamby Ambikiarajah, David J. Webb, and Gang-Ding Peng, "A Fast Response Intrinsic Humidity Sensor Based on an Etched Singlemode Polymer Fiber Bragg Grating," Sensors and Actuators A: Physical, 203, 107-111, 2013

[18] C. Zhang, W. Zhang, D.J. Webb, G.-D. Peng, “Optical fiber temperature and humidity sensor", Electron. Lett. Vol.46 p. 643, 2010. 